\title{
Review of: "Full-length NLRP3 forms oligomeric cages to mediate NLRP3 sensing and activation"
}

\author{
$\mathrm{Cu}^{1}$ \\ 1 Zhejiang University
}

Potential competing interests: The author(s) declared that no potential competing interests exist.

NLRP3 is a critical intracellular sensor that detects a broad range of pathogens and damage-associated signals, resulting in the formation and activation of the NLRP3 inflammasome. Although the studies on NLRP3 is more extensive than other NLR family members (i.e. NLRP6, NLRC4, NLRP1), the structure of NLRP3 has not been fully understood. In this study, the authors discovered a novel 12-16 mer double ring cage structure, in which PYD and LRR domains paly essential roles. It explains the reason why the previous study that usually uses PYD-lacking mutation for structure analyses does not work properly on NLRP3. The authors performed NLRP3 overexpression, purification, Negative-staining, cryo-electron microscopy, in vitro lipid blot assay, and immunofluorescence to find out that resting NLRP3s are mainly localized on membranes in a double ring cage structure to avoid premature activation. Upon LPS priming and Nigericin stimulation, this cage structure is also required for TGN dispersion which traffics to the MTOC and finally triggers NLRP3 inflammasome assemble. A perfect logic could be seen here. Overall, the experimental plan is theoretically perfect, the writing is rigorous, and the findings provide a striking mechanistic basis for NLRP3 inflammasome trafficking and activation. However, some bold claims are made with unclear support which might need following works to supplement.

Specific Comments:

1. What is the peripheral membrane that inactivated NLRP3 double ring combined with? Is it the trans face of Golgi? Is it possible to test the interaction (IF/Western blot) among NLRP3, TGN38, and NEK7 in iBMDMs from resting to stimulation state?

2. If the polybasic region which binds to TGN is necessary for NLRP3 double ring structure, then how could the double ring structure be detected in cytoplasm? Does it fall down from membrane after the double ring formation?

3. Please compare the size of NLRP3 cage in iBMDMs and HEK293T cells by running naive PAGE gel together and add the data in Figure 3h.

4. In "Full-Length NLRP3 exists as large oligomeric structures" part, why did the author use ADP+MCC950 but not use ADP or MCC950 to treat separately?

5. In Extended Data Fig. 1a, why the flag-cleaved NLRP3 has a bigger structure? Could the authors show 
some repeated results?

6. Is it possible to use the NLRP3-dPYD mutant as a control to observe the negative staining results without double ring structure?

7. In Extended Data Fig. 1b, please explain whether the group of dATP+MCC950, ADP, and ADP+MCC950 is required.

8. In Extended Data Fig. 2a, why the 2D classification pictures of 6-/7-/8-fold double ring cages are counted as 7-/8-/9- mers?

9. Is the percentage of 6-/7-/8-fold double ring cages different between ADP+MCC950, ATP, no nucleotide treatment?

10. Could the author explain the dispersion state of 6-fold structure in Extended Data Fig. 2b?

11. Please explain the 40-70kDa strips in the 1uM NLRP6 ${ }^{\text {PYD-NACHT }}$ on Extended Data Fig. 5b. Are they meaning that other NLRP3 inflammasome components (i.e. Caspase/ASC) are included here?

12. In Figure 3i, please explain the difference between NLRP3 cage and NLRP3 monomer. And what is the meaning of labeling WT and NLRP3dPYD together in the 2 nd and 3rd row on the right side of the figure. From my perspective, the mutant that only has Polybasic region mutated without removing the PYD should also be used as a control here.

13. Please add unstimulating controls (without LPS priming) in all the immunofluorescent results of Figure 4 and Extended Data Fig. 6.

14. Please provide the results regarding only LPS priming without Nigericin stimulation in Extended Data Fig. 6a of g-tubulin and ASC staining.

15. In Extended Data Fig. 6b-d, please stain the 58K Golgi and g-tubulin at the same time to see if it is LPSpriming that makes Golgi transport the NLRP3 double ring structure to MTOC would be appreciated.

16. Please provide the original data in Figure $4 \mathrm{I}$ and detailed information about how many repeat groups used here as well as adding statistical analysis.

Minor Issues:

1. More details should be included in the introduction, for instance the information of TGN dispersion with NLRP3 activation and the trafficking of TGN vesicles to MTOC.

2. Please explain the reason why the NLRP3 is required to be combined with Phosphorylated Ptdlns.

3. Where is the data of cryo-EM data in sentence "We collected cryo-electron microscopy (cryo-EM) data on these samples. 2D classification of these datasets revealed that dATP gave most homogenous complexes with finer details......"?

4. Please add MW label (KDA) on the Western blot in Extended Data Fig. 3h.

5. The sentence here is confusing, “Local resolution estimation indicated lower resolutions at the NACHT domains, likely indicative of flexibility, as compared to the better resolved LRRs". According to my understanding, the NACHT resolution should be higher. 
6. "In addition, they assume an inactive conformation almost identical to those in the autoinhibited NLRC4 and the NLRP3-NEK7 complex structures", here "they" should be we?

7. Is the "per se" need to be deleted in this sentence, “... (Fig. 3m), indicating that the membrane localization per se is not sufficient and that the cage-forming interfaces are also required"? 